\title{
Chronic pain and whiplash associated disorders: Rehabilitation and secondary prevention
}

\author{
Prof. Dennis C. Turk, Ph.D.*
}

$E_{s}^{s}$ stimates suggest that over 60 million A mericans suffer from some type of chronic pain sufficient to impact significantly on their lives (1). $\mathrm{N}$ ot only does chronic pain adversely affect pain sufferers' physical and psychological well-being, it also costs billions of dollars to society in lost productivity, health care expenditures, disability compensation, and tax revenues. The health care costs incurred by chronic pain patients range from $\$ 500$ to $\$ 35,400$, with the average being $\$ 13,284$ annualIy (2). These expenditures do not include the costs of surgical procedures. Surgical procedures add substantially to health care expenses. For example, the most prevalent complaint of patients (40\%-60\%) is some form of back pain. The cost for lumbar surgery is approximately $\$ 15,000$ with a total of almost $\$ 325$ billion annually spent on surgery for chronic back pain patients alone (3). Furthermore, heal th care consumption following initial surgery may increase for some patients due to iatrogenic complications. Significant complications requiring additional surgery are relatively common. Research indicates that up to a third of the operations performed for back pain result from pain secondary to an original surgical procedure designed to alleviate pain (4).

Chronic pain is especially difficult to treat successfully. Despite major advances in knowledge of sensory physiology, anatomy, and biochemistry along with the development of potent analgesic medications and other innovative medical and surgical interventions, relief for many pain sufferers remains elusive. The lack of satisfactory outcomes from the traditional medical, pharmacological, and surgical approaches was an impetus to the development of specialty treatment facility - Multidisciplinary Pain Centers (MPCs) to treat patients with recalcitrant pain problems. 0 ver 400 pain treatment facilities have been established in the U nited States with an additional 1000 worldwide. A wide range of treatments is provided at these facilities. The estimated average cost for an outpatient treatment by pain specialists is $\$ 8,100$ (5). Extrapolating from available survey data, I estimate that over $\$ 1.5$ billion is spent annually on treatment at specialized facilities. The largest majority of patients treated at M PC s have pain of musculoskeletal origin. Up to $40 \%$ report that their symptoms began following a trauma such as a motor vehicle accident (M VA).

Rehabilitation programs - Interdisciplinary Pain Rehabilitation Programs (IPRPS) that address pain reduction, functional restoration, and improvement in quality of life as alternatives to treatments designed primarily to substantially reduce, if not eliminate pain, have received a great deal of attention. One fundamental concept common to rehabilitation of chronic pain patients is the understanding that patients with complex pain problems are best served by a team of specialists with different health care backgrounds. The report of pain is not just the result of body damage but has psychological and environmental origins as well. Thus, IPRPs treat not only the experience of pain but also associated patient distress, dysfunction, and disability associated with chronic pain. A Ithough there is no standard protocol for IPR Ps, they all tend to include a core group of health professionals including physicians, physical therapists, and psychologists.

It is reasonable to consider the question of the effectiveness of IPRPS. A sking whether IPR Ps are generally effective, however, may be an inappropriate way to phrase the question of whether they are worth while. It might be more appropriate to broaden the question to ask how effective are pain treatment facilities compared to alternative treatments and on what outcome criteria. That is, we can ask how effective are IPRPs compared to alternatives such as surgery, neuroaugmentation procedures, and long-term drug therapies on reduction in pain, reduction in medication and health care utilization, increased physical activity, closure of disability claims, and return to work.

Several reviews and meta-analyses on treatment outcome studies have evaluated the clinical and cost effectiveness of IPRPS (6-8). Despite the recalcitrance of the pain problems of the patients treated, the outcome data generally support the efficacy of IPRPs on a range of criteria including pain reduction, improvements in functional activities (e.g., activities of daily living, return to work), alleviation of depression, lessening of health care consumption, and termination of disability claims. IPR Ps and more conventional measures have about the same effect on alleviating pain. It is important to acknowledge, however, that none of the tools - drugs, regional anesthesia, surgery, and rehabilitation - currently available consistently eliminates pain in all pain sufferers.

IPRPS appear to be more effective than pharmacological and surgical approaches in significantly reducing health care consumption, resulting in the closure of disability claims, increasing functional activities, and helping patients return to

* D epartment of A nesthesiology, U niversity of W ashington, Seattle, W ashington, U SA

Correspondence: Dr DC Turk, D epartment of A nesthesiology, U niversity of W ashington, H SB 1425, BB-1469 H ealth Sciences, Box 356540, Seattle, Washington 98195, U SA. Telephone 206-616-2626, fax 206-543-2958, e-mail turkdc@u.washington. edu. 
work. Interestingly, the improvements observed at IPR Ps have been achieved along with concomitant reductions in opioid medication consumption. It is particularly noteworthy that reductions in pain observed occur in conjunction with lessening analgesic use.

IPRPs have also been shown to be more cost-effective than patient education and physical therapy alone, surgery, neuroaugmentative procedures (i.e., spinal cord stimulators, implantable analgesic pumps), and prescriptions for long-term opioid medication (9). O ne factor contributing to the comparative cost benefits for IPR Ps, in contrast to neuroaugmentative modalities and long-term opioid therapy, is that no additional medical monitoring of patients treated at IPR Ps is required.

To illustrate the differences in treatment outcomes between IPRPs that focus on rehabilitation and conventional alternatives, I note that, based on a meta-analysis of 65 published studies of the outcomes of IPRPs that included 3089 patients, $45 \%-65 \%$ of patients treated at IPR Ps return to work following treatment (6). These results can be compared to studies reporting that only $20 \%$ of patients return to work following surgery for pain and $25 \%$ return to work following implantable pain control devices. Studies have reported that a significant percentage of chronic pain patients treated with surgery report that their pain is worse following surgery (10). Subsequent operations do not guarantee resolution of pain with some studies acknowledging the poor results achieved for reoperations.

There are no randomized control trials demonstrating the effectiveness of long-term opioids and little evidence at all to support the success of long-term use of opioids in improving patients' functional outcomes including return to work. M oreover, in contrast to alternatives such as surgery and neuroaugmentative procedures that report complications of treatment ranging up to $50 \%$, there are also no reported iatrogenic consequences for IPR Ps. The results of the meta-analysis mentioned (6) above indicate that following treatment at IPRPs, patients required one-third the number of surgical intervention and hospitalizations compared to patients treated by alternative medical and surgical care. Furthermore, treatment at IPRPs resulted in closure of disability claims for one-half of those receiving disability at the time of treatment. Even at long-term follow-up, patients who are treated in pain rehabilitation programs appear to function better than $75 \%$ of chronic pain patients treated by alternative treatment approaches.

Based on extrapolations from the data from 3089 patients treated at IPRPs included in one meta-analysis (6), I estimate that savings in excess of $\$ 20$ million would be achieved based on reductions in health care consumption and indemnity costs during the first year following treatment, even after factoring the cost of treatment at IPR Ps. Considering the average age of the patients treated by pain specialists is 45 , the anticipated savings until age 65 would exceed $\$ 248$ million. If I use the same assumptions for the estimated 175,000 patients treated at IPRPs in the U nited States, then the financial savings would exceed $\$ 11$ billion in the first year following treatment alone.

M ost IPR Ps include a broad range of components within a single rehabilitation package. Further research is needed to isolate the shared components of various successful treatment programs. There are no data available identifying the characteristics of patients who would most likely benefit from any of the pain treatment methods available. What are needed are studies that answer the question: what treatments delivered in what ways are most effective for patients with what set of characteristics? Successful answers to this question will permit more clinically effective and cost effective ways to treat the difficult population of patients with chronic pain.

We know that treatment of chronic pain patients is difficult and costly. This insight should give greater impetus to prevention. N amely, which people are at risk for chronic pain and disability following initial symptom onset and what types of interventions can be developed and implemented to prevent those at risk individuals for going on to chronicity. These questions seem particularly appropriate when we consider people who have suffered whiplash injuries following M VA s.

Prospective studies have shown that between $24 \%$ and $62 \%$ (11-12) of whiplash victims initially presenting to a trauma unit or physicians' offices continue to be symptomatic for extended periods following MVA s. Epidemiological research indicates a 33\% risk of continued symptoms at approximately three years following an M VA (13). Thus, it appears to be reasonable to estimate that between $24 \%$ and $33 \%$ of patients will have chronic physical symptoms over a year after sustaining a whiplash injury.

W hat factors predict chronicity? If we had an answer to this question we might be able to design a program and intervene selectively to prevent the development of chronic pain and disability. Research evaluating factors predictive of chronicity in whiplash associated disorders (WA Ds) symptoms are equivocal. A number of variables have been reported to be significant predictors of chronic WA D symptoms including physical (e.g., multiple injuries and initial symptoms [11-14]), demographic (sex, age, marital status [15-17]), and accident-related factors (e.g., being in collision involving fatality or severe injury [14-16]). Some studies failed to replicate these findings (18-19). Other factors immediately following the accident such as neck pain or stiffness, headache, intercapsular pain, and differences in speed between motor vehicles were not predictive of chronicity of symptoms.

Early reports have suggested that pre-existing psychopathology or neurotic features might be the underlying mechanisms for unremitting WAD problems (20-21). Preexisting emotional disturbances, coupled with medical complications does appear to predict chronicity of WA Ds at least for some individuals. H owever, the results of other studies implicate psychological symptoms as concomitants rather than precursors to chronic symptoms after WA D. Initial reaction to the accident, rather than the pre-existing psychological status, has been shown to predict chronicity (11-22). However, preexisting psychological status may predispose some individuals to chronic emotional disturbances following an MVA. For example, acute emotional distress has been shown to be related to pain severity one month following an M VA (23). These results suggest that an early intervention to help whiplash victims adjust emotionally may reduce the likelihood of developing long-term WA D symptoms and disability.

Research investigating the evolution of chronic pain due to musculoskel etal injury suggests that initial emotional reactivity, particularly fear of reinjury and subsequent activity avoidance, contribute significantly to unremitting pain and 


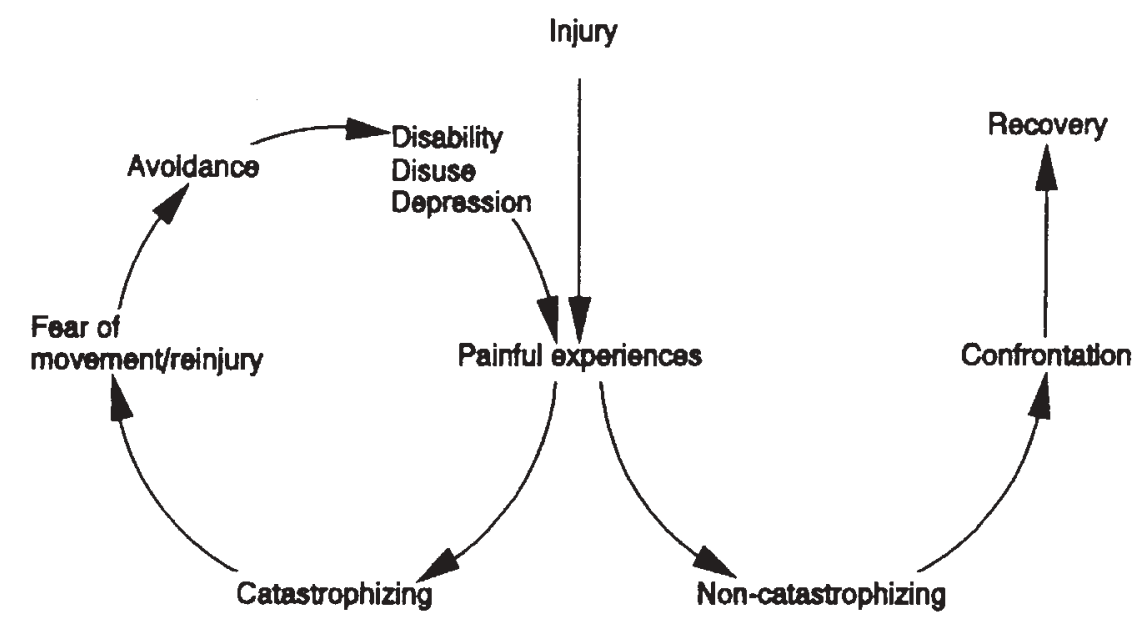

Figure 1 C ognitive-Behavioral M odel of F ear of M ovement/(Re)Injury from VLAEYEN ET AL., 1995

persistent disability (24). Based upon this model, early interventions targeting reduction of fear and subsequent avoidance of activity for injury victims have been shown to prevent chronic musculoskeletal pain and disability resulting from back injury. The model also suggests that people prone to negative emotional responses are at risk of experiencing elevated levels of fear and anxiety and consequently may be predisposed to develop WA Ds. Thus it appears that anxiety, fear of pain, and fear of injury or reinjury may be important contributors to chronicity.

The underlying premise of the anxiety-based model is the central role of fear of pain and in particular the fear that physical activity will cause pain and (re-)injury. Thus, fear of pain may lead to long-term avoidance of activities. The deconditioning that results from such avoidance may lead to greater disability.

Exposure to activity is adaptive and necessary to reduce fear and promote recovery after the injury is resolved. In contrast, avoidance of feared activities leads to the maintenance or exacerbation of fear and inadequate corrective feedback. Further, avoidance of both social and physical activities may result in maladaptive physical and psychological consequences that augment disability. KORI ET A L. (25) introduced the term 'kinesiophobia' (fear of movement) for the condition in which a patient has "an excessive, irrational, and debilitating fear of physical movement and activity resulting from a feeling of vulnerability to painful injury or reinjury".

The schematic presentation of the cognitive-behavioral model of fear of movement and how it creates the vicious circle of chronic pain is described in the figure (24). Certain cognitive response (e.g., catastrophizing) in response to painful experience following injury is augmented in people predisposed with high levels of negative affectivity, leading to fear of movement. This fear of movement leads the person to avoid activities that he or she believes will aggravate the injury and cause continued pain. A voidance of activities not only serves to 'prevent' further painful experience but also promotes disability and deconditioning due to disuse of muscles. In the short run, this avoidance of activity may lead to a decrease in pain, reinforcing catastrophizing, fear responses, and continued avoidance of movement. It has been shown that painrelated fear is associated with escape by avoidance of activity and self-reported disability.

There are some preliminary data to support the fear avoidance model with chronic back pain patients. My colleagues and I have recently begun a study that will evaluate the effectiveness of an exposure-based secondary prevention treatment that was derived from the fear-avoidance model with people suffering Level 1 and 2 WA D s within the first three months of symptom-onset following an M VA. In this study we will evaluate the effectiveness of the exposure model in preventing chronic pain and disability in those whose symptoms have not resolved by three months. This study will permit us to evaluate the utility of the model in preventing chronicity.

In summary, a substantial body of literature supports the assertion that IPRPs are effective in reducing pain, use of opioid medication, reduced use of health care services, increasing activity, returning people to work, and closing disability claims. Comparisons of IPR Ps with alternative pharmacological and surgical interventions suggest that the rehabilitation programs are more effective. $\mathrm{N}$ ot only do IPRPS appear to be clinically effective; they also appear to be cost effective with the potential to provide substantial savings in health care and disability payments. These results are especially impressive when we realize that treatment at IPR Ps targets patients with the most recalcitrant problems (i.e. long duration, failure of many previous therapies). Although the effectiveness of IPRPS for WAD sufferers has not specifically been evaluated, there seems no reason to believe that the results would not be comparable. However, regardless of the success of IPRPs in treating patients with chronic pain, it is reasonable to consider the potential clinical and costs benefits of preventing chronicity. I described an anxiety-based model and proposed the potential of this model to prevent chronicity. The model is heuristic and awaits systematic evaluation with WAD sufferers. 


\section{REFERENCES}

1. BONICA JJ. General considerations for chronic pain. In: BonICA JJ, LOESER JD, Cha pman CR, Fordyce WE, eds. The M anagement of Pain, 2nd ed. Philadel phia: Lea \& Febinger, 1990; 180-96.

2. BELL G, KIDD D, NORTH R. Cost-effectiveness analysis of spinal cord stimulation in treatment of failed back surgery syndrome. J Pain Symp M anag 1997; 13,286-95.

3. FRYM OYER J, DURETT C. The economics of spinal disorders. In: FRYM OYER J. ed. The A dult Spine, 2nd ed. Philadel phia: LippincottRaven, 1997: 143-150.

4. Long DM, Filtzer DL, Ben Debba M, Hender N H. Clinical features of the failed-back syndrome. J N eurosurg 1988; 69:61-71.

5. Market Data Enterprises. Chronic Pain Management Programs: A M arket A nalysis. N ew York: Valley Stream, 1995.

6. FLOR H, FYDRICH T, TURK D. Efficacy of multidisciplinary pain treatment centers: a meta-analytic review. Pain 1992; 49; 221-30.

7. Morley S, ECCLESTON C, W ILlia MS A. Systematic review and metaanalysis of randomized controlled trials of cognitive behaviour therapy and behaviour therapy for chronic pain in adults, excluding headache. Pain 1999; 80:1-13.

8. Guzman J, Esmail R, Karjalainen K, Malmivaara A, Irvin E, BOM BA RDIER C. M ultidisciplianry rehabilitation for chronic low back pain: systematic review. BM J 2001; 322:1511-16.

9. TURK DC, O KIFUJI A. Treatment of chronic pain patients: clinical outcomes, cost-effectiveness, and cost-benefits of multidisciplinary pain centers. C rit Rev Phys M ed Rehabil Med 1998; 10:181-208.

10. Franklin GM, Haug J, HeYer NJ, et al. O utcome of lumbar fusion in Washington state workers' compensation. Spine 1994; 19:1897-904.

11. Gargan M, Bannister G, Main C, et al. The behavioural response to whiplash. J Bone Joint Surg Br 1997; 79:523-6.

12. Pennie B, A gambar L. Patterns of injury and recovery in whiplash. Injury 1991; 22:57-9.
13. Freeman M D, Croft A C, Rossignol A M. "W hiplash associated disorders: redefining whiplash and its management" by the Q uebec Task Force. A critical evalution. Spine 1998; 23:1043-9.

14. Spitzer W O, Skovron M L, Salmi LR, et a L. Scientific monograph of the Q uebec Task Force on W hiplash-A ssociated Disorders: redefining "whiplash" and its management. Spine 1995; 20:1S-73S.

15. DeANS G, MagalLIARD J, KeRr M, ET AL. N eck pain - a major cause of disability following car accidents. Injury 1987; 19:10-2.

16. DoLINIS G. Risk factors for 'whiplash' in drivers: a cohort study of rear-end traffic crashes. Injury 1997; 28:173-9.

17. Sturzenegger M, Radanov BP, Di Stefano G. The effect of accident mechanisms and initial findings on the long-term course of whiplash injury. J N eurol 1995; 242:443-9.

18. HARVEY A G, BRYANT RA. Predictors of acute stress following motor vehicle accidents. J Trauma Stress 1999; 12:519-25.

19. HILDINGSSON C, TOOLANEN G. O utcome after soft-tissue injury of the cervical spine. A prospective study of 93 car-accident victims. A cta O rthop Scand 1990; 61:357-9.

20. GAY J, A ввот K. Common whiplash injuries of the neck. JA M A 1953; 152:1698-704.

21. HODGE JR. The whiplash neurosis. Psychosomatics 1971; 12:245-9.

22. Drottning M, StafF P, LeVIN L, et AL. A cute emotional response to common whiplash predicts subsequent pain complaints. A prospective study of 107 subjects sustaining whiplash injury. N ordic J Psychiatry 1995; 49:292-99.

23. Mayou R, Bryant B, Duthie R. Psychiatric consequences of road traffic accidents. BMJ 1993; 307:647-51.

24. Vlaeyan JW, Kole-Snijders A M, Boeren RG, et al. Fear of movement/(re)injury in chronic low back pain and its relation to behavioral performance. Pain 1995; 62:363-72.

25. KORI S, MILLER R, TODD D. Kinisophobia: a new view of chronic pain behavior. Pain M anag 1990; Jan/Feb:35-43.

\section{A II costs are in U S dollars.}

This material was presented at the International Congress on W hiplash A ssociated Disorders, Berne, Switzerland, M arch 8 to 10, 2001 . The paper appeared originally in the book "W hiplash A ssociated D isorders" - medical, biomechanical and legal aspects, published by Staempfli Publishers Ltd, Berne 2002. The paper is published in N orth A merica in Pain Research \& M anagement with the permission of Staempfli Publishers Ltd. 


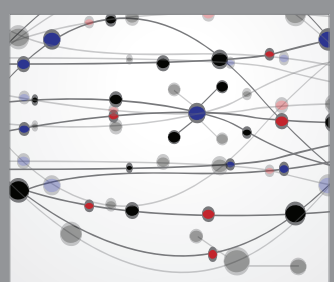

The Scientific World Journal
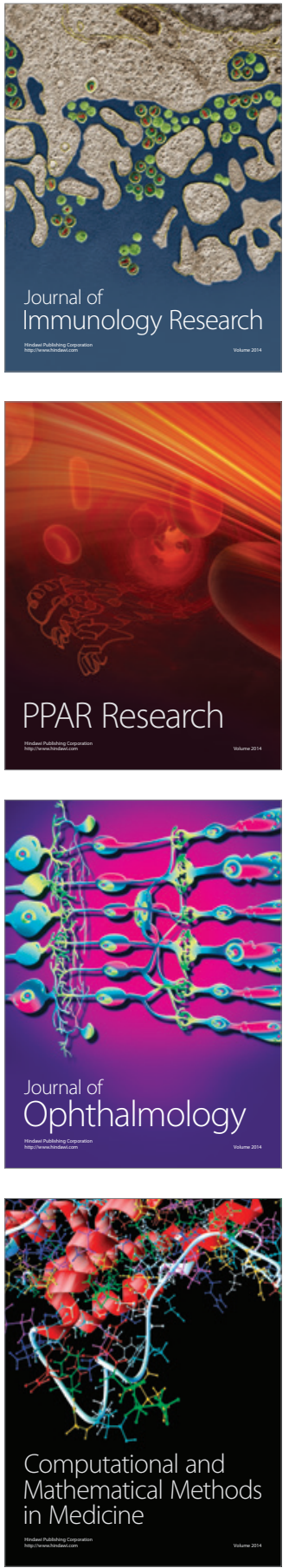

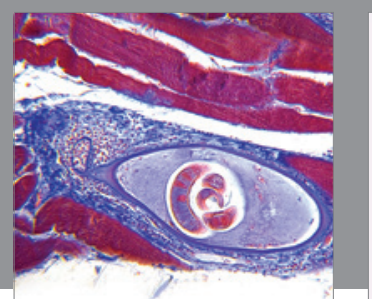

Gastroenterology Research and Practice

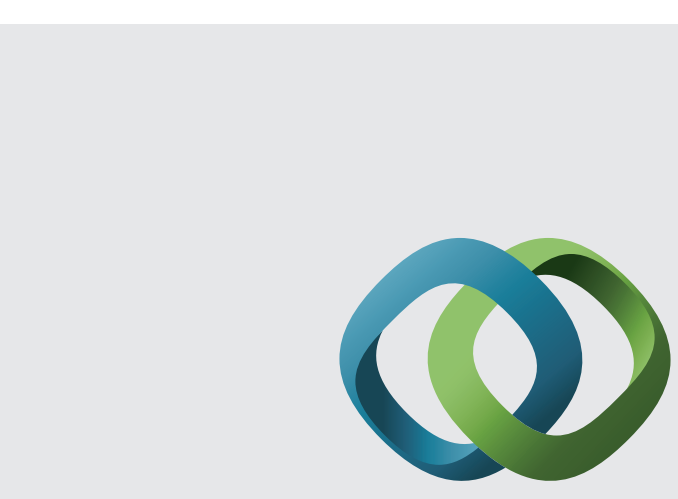

\section{Hindawi}

Submit your manuscripts at

http://www.hindawi.com
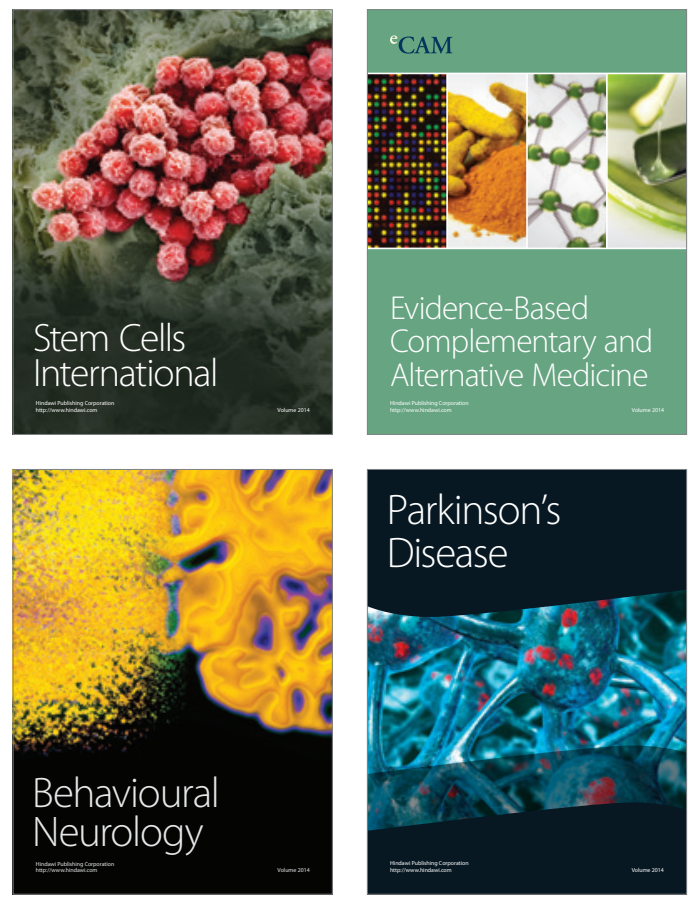
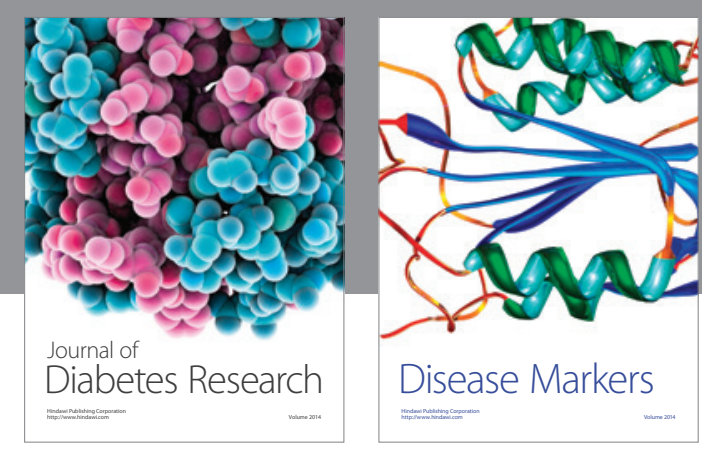

Disease Markers
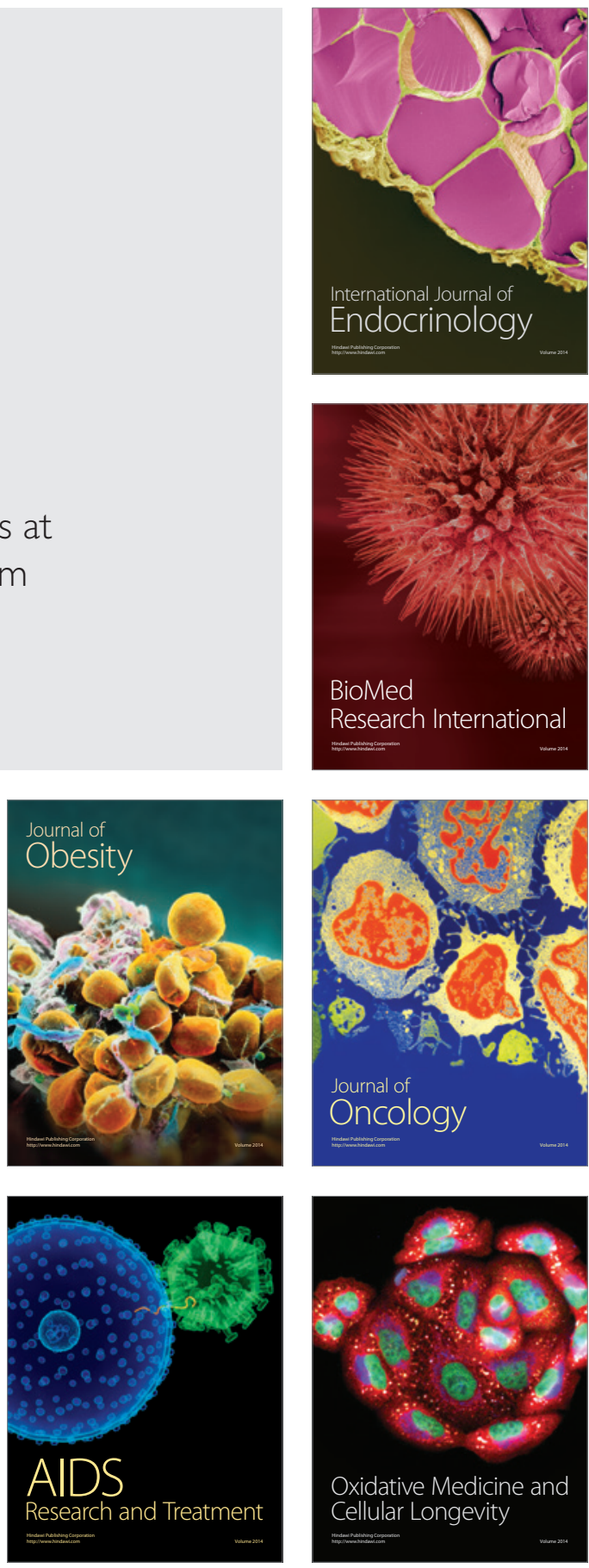